\title{
Cerebrospinal Fluid Involvement in Mantle Cell Lymphoma
}

Riccardo Valdez, M.D., Steven H. Kroft, M.D., Charles W. Ross, M.D., Bertram Schnitzer, M.D., Timothy P. Singleton, M.D., LoAnn C. Peterson, M.D., William G. Finn, M.D.

Department of Pathology, University of Michigan (RV, CWR, BS, TPS, WGF), Ann Arbor, Michigan; University of Texas Southwestern Medical School (SHK), Dallas, Texas; Northwestern University Medical School (LCP), Chicago, Illinois

Mantle cell lymphoma (MCL) is an aggressive neoplasm that is incurable by standard therapy. Patients often present with high-stage disease (Stages III-IV) and frequently have involvement at multiple extranodal sites. Although the gastrointestinal tract, spleen, lung, pleura, bone marrow, and peripheral blood are among the most commonly involved tissues, MCL may also disseminate to so-called sanctuary sites including the central nervous system. Despite this, current clinical evaluations do not routinely include assessment of the cerebrospinal fluid (CSF) for lymphomatous infiltrates at presentation, and moreover, only few authors have specifically examined CSF involvement in MCL. In this study, we reviewed the medical records of 108 patients with MCL seen at three centers over a 5-year period to determine the rate of CSF sampling and the frequency of CSF involvement by MCL. The clinical and cytologic characteristics associated with CSF involvement were also studied. Central nervous system (CNS) signs and/or symptoms prompted CSF sampling in $25 / 108$ patients $(23 \%)$. Specific radiographic abnormalities were present in 9/25 patients (36\%). CSF involvement by MCL was identified in 10 of the 25 CSF-sampled patients $(40 \%)$ by morphology (3 patients), flow cytometry alone (1 patients), or both (6 patients). The CSF-positive cases included two blastoid variants. The CSF cytology of the nine morphologically positive cases was pleomorphic, and prominent cytoplasmic granules were observed in two cases. The overall rate of CSF involvement by MCL among the cohort was $9 \%$, which

Copyright (C) 2002 by The United States and Canadian Academy of Pathology, Inc.

VOL. 15, NO. 10, P. 1073, 2002 Printed in the U.S.A.

Date of acceptance: June 27, 2002.

Address reprint requests to: Riccardo Valdez, M.D., Department of Pa thology, University of Michigan, 1301 Catherine Street M5242 Medical Sciences Building I, Ann Arbor, MI 48109-0602; e-mail: rcdovldz@umich.edu; fax: 734-936-2756.

DOI: $10.1097 / 01 . M P .0000030088 .28684 .5 B$ is comparable to that reported in other selected studies examining this issue.

KEY WORDS: Central nervous system, Cerebrospinal fluid, Mantle cell lymphoma, Non-Hodgkin's lymphoma.

Mod Pathol 2002;15(10):1073-1079

Mantle cell lymphoma (MCL) is a distinctive type of non-Hodgkin's lymphoma composed of small-tomedium-sized lymphocytes that coexpress pan-Bcell antigens and the pan-T-cell antigen, CD5. The chromosome translocation $\mathrm{t}(11 ; 14)$ (q13; $\mathrm{q} 32)$ results in activation of the PRAD-1/cyclin-D1 gene (1-3) and further characterizes MCL. Recent studies suggest that MCL accounts for approximately 5-6\% of all non-Hodgkin's lymphomas in the United States and Europe, and despite its histologic resemblance to low-grade B-cell lymphomas, MCL is an aggressive disease with a poor prognosis. The median survival of typical MCL patients is approximately 43 months $(2,4)$. Patients with the blastoid variant of MCL, which features high mitotic activity and morphologic resemblance to lymphoblastic lymphoma, may have an even poorer prognosis $(2,4-6)$.

MCL patients usually present with multifocal disease, including frequent lymphomatous involvement at extranodal sites such as the gastrointestinal tract, spleen, liver, Waldeyer's ring, lung, and pleura. Involvement of more than one extranodal site at presentation occurs in $\leq 37 \%$ of patients (4). In addition, approximately $80 \%$ of patients have disease in the bone marrow, and $\leq 35 \%$ have leukemic involvement of the peripheral blood at diagnosis (7). Response to therapy is often short-lived, and relapses are common. Despite this aggressive behavior, clinical evaluations of MCL patients do not routinely include assessment of the cerebrospinal fluid (CSF) as they do in lymphoblastic and Burkitt-type lymphoma protocols. Moreover, unlike the case with other aggressive lymphomas, only 
very few studies have specifically examined CSF involvement in MCL $(14,16)$.

In this study, we retrospectively studied a cohort of 108 patients with MCL seen at three separate tertiary care centers to determine the rate of CSF sampling and the frequency of CSF involvement by MCL. In addition, we examined the clinical characteristics and cytologic features associated with CSF involvement by MCL.

\section{MATERIALS AND METHODS}

The pathology files of the University of Michigan Health System (Ann Arbor, MI), University of Texas Southwestern Medical School (Dallas, TX), and Northwestern University Medical School (Chicago, IL) were searched for all cases of MCL seen between 1993-1998. All cases with either classic or consistent lymph node morphology by routine hematoxylin and eosin stains and supportive immunoperoxidase and/or flow-cytometric immunophenotyping results were selected for the study. MCL involvement at extranodal tissue sites in two cases (testis and breast) was confirmed by immunohistochemical and flow cytometric immunophenotyping. These studies were carried out using previously described methods $(8-12)$.

From a total of 113 cases meeting published diagnostic criteria for MCL (13), adequate medical records were available for 108 patients. The clinical, radiographic, and laboratory records of these 108 patients were reviewed for the following: (1) evidence of CSF sampling for any reason either before, at, or after diagnosis and (2) any clinical, laboratory, and/or radiographic evidence of CSF/central nervous system (CNS) involvement by MCL at any time during the course of disease. Twenty-five patients from whom the CSF was sampled at some time after diagnosis were identified among the cohort; no patients had CSF sampling before the time of diagnosis. All accessible CSF data from these 25 patients (including chemistries, cell counts and differentials, routine Wright-stained cytocentrifuge smears, and flow-cytometric immunophenotyping findings) were analyzed, and the results were correlated with available clinical, radiographic, and other laboratory data. Cytologic examination of the CSF was limited to Wright-stained smears because these smears were uniformly available for review in all CSF-sampled cases and also because this is the most frequently used stain preparation for CSF examination in hematology and flow cytometric laboratories. Patient medical record reviews were performed independently by a pathologist at each participating hospital, and the data were subsequently compiled and analyzed by two of the authors (RV, WGF). Similarly, all CSF smears were first independently reviewed and diagnosed at each of the participating hospitals and then rereviewed centrally by five of the authors (RV, TPS, CWR, BS, WGF). The study was closed at the end of 1998 in an effort to obtain all possible follow-up information and to allow for the central data collection and review.

\section{RESULTS}

CSF was sampled by routine lumbar puncture (LP) in 25 of the 108 (23\%) MCL patients in our cohort (Table 1). This group consisted of 17 men and 8 women with a mean age of 58.1 years at the time of diagnosis with MCL (range, 44-78 y). Twenty-two of these 25 patients (88\%) presented with Stage IV disease, whereas 2 of 25 (8\%) were Stage I at presentation. Stage was not available for one of the CSF-sampled patients. At the time of diagnosis, 24 of 25 (96\%) patients had typical diffuse MCL morphology on lymph node biopsy, and one patient presented with the blastoid variant of MCL. The patient with blastoid MCL was one of two patients who presented with Stage I disease. Clinical CNS signs and/or symptoms (including headache, fever, weakness, paresthesias, mental status changes, visual disturbances, and/or specific cranial nerve palsies) prompted CSF sampling in all 25 patients. In addition, radiographic data were available for 17 of 25 patients, and abnormalities of the CNS were observed in 9 of 17 (53\%) at the time of presentation with clinical CNS signs and/or symptoms.

Ten of the 25 patients (40\%) who underwent LP were positive for MCL involvement of the CSF by morphology (3), flow-cytometric immunophenotyping (1), or both (6). This subgroup of CSFpositive patients consisted of 6 men and 4 women with a mean age of 59.4 years (range, $44-78$ y) and

\begin{tabular}{lc} 
TABLE 1. Characteristics of 25 CSF-Sampled MCL \\
Patients \\
\hline No. patients sampled by CSF \\
Age at diagnosis (y) \\
$\quad$ Range & $25 / 108$ \\
Mean & 54.1 \\
Male/Female & $17 / 8$ \\
Stage at diagnosis & 2 \\
I & 22 \\
IV & 1 \\
Unknown & 24 \\
Histologic type at diagnosis & 1 \\
Typical & \\
Blastoid & 25 \\
Reason for CSF sampling & 9 \\
Clinical signs/symptoms & \\
Radiologic lesion & $10 / 25(40)$ \\
CSF involved by MCL, $n$ (\%) & $10 / 108(9)$ \\
CSF-sampled cohort only & \\
Entire MCL cohort &
\end{tabular}

CSF, cerebrospinal fluid; MCL, mantle cell lymphoma. 
included both patients initially diagnosed with Stage I disease (including one patient with the blastoid variant; Table 2). Furthermore, the $10 \mathrm{CSF}$ positive cases also included one patient with typical MCL at diagnosis who developed a blastoid transformation in the bone marrow concomitant with CSF involvement by MCL. Radiographic studies of the CNS were performed in 6 of the 10 patients with CSF involvement by MCL, and specific lesions were found in 5 of these patients (83\%). Of note, 4 of the 25 CSF-sampled patients with observed radiographic abnormalities [orbital mass (2), intracerebral bleed (1), and nasal/maxillary mass (1)] did not have documented involvement of the CSF by MCL despite multiple LPs. The clinical CNS signs and symptoms exhibited by the 10 CSF-positive patients were similar to those of the 15 CSF-negative patients. The mean and median times from initial MCL diagnosis to CSF involvement were 22.6 months and 15.5 months, respectively (range, 0-96 mo).

Concomitant with CSF positivity for MCL, six patients were leukemic, eight had infiltration of the bone marrow, one patient had involvement of the testis, and one had MCL in the breast. Only 1 of the 10 CSF-positive patients had no evidence of disease outside of the CNS. This was a patient with blastoid MCL who presented with Stage I disease 21 months before an apparent primary CNS relapse (cerebropontine angle mass).

Routine CSF laboratory data were available for all 10 CSF-positive patients and are displayed in Table 3. The mean CSF white blood cell count (white blood cell count) was 1307 per cubic millimeter (range, 1-4950 per cubic millimeter), with the high- est white blood cell count recorded in the patient with the blastoid MCL and cerebropontine angle mass (4950 per cubic millimeter). In all cases, the CSF white blood cell population was composed predominantly of lymphocytes (range, 68-100\%) with the remaining cells being mostly histiocytes. Analysis of the CSF chemistries revealed an elevated CSF protein level in all but two patients; CSF glucose levels were decreased in five patients, within normal limits in two, and elevated in three.

Flow-cytometric immunophenotyping of the CSF was performed in 7 of the 10 patients and showed monoclonal CD19 and CD20 positive B-cell populations that coexpressed CD5 in all seven cases. The diagnosis of CSF involvement by MCL in the three patients for whom flow cytometry was not performed was made solely by cytologic assessment. MCL in the CSF was diagnosed by flow cytometry alone in one case in which there was an insufficient number of cells on the cytocentrifuge smear for complete evaluation.

In the nine morphologically positive cases, the appearance of the MCL cells on the examined Wright-stained cytocentrifuge CSF preparations was pleomorphic and variable. In six of nine cases, the CSF contained a mixture of small-to-large lymphoid cells with irregular nuclei and inconspicuous nucleoli, resembling leukemic MCL (Fig. 1). In the other three cases, including one with typical MCL lymph node histology and the two blastoid variants, the malignant cells were predominantly large with slightly irregular nuclei, dispersed chromatin, and prominent nucleoli, resembling other blastic-type proliferations involving the CSF or other body fluids (Fig. 2). The amount and

TABLE 2. Clinical Characteristics of $10 \mathrm{MCL}$ Patients with CSF Involvement

\begin{tabular}{|c|c|c|c|c|c|c|c|}
\hline \multirow{2}{*}{$\begin{array}{l}\text { Patient } \\
\text { No. }\end{array}$} & \multirow{2}{*}{$\begin{array}{l}\text { Age at } \\
\text { Diagnosis } \\
\text { (y)/Sex }\end{array}$} & \multirow{2}{*}{$\begin{array}{l}\text { Stage at } \\
\text { Diagnosis }\end{array}$} & \multirow{2}{*}{$\begin{array}{l}\text { Histologic } \\
\text { Type }\end{array}$} & \multicolumn{2}{|c|}{ Reason for CSF Sampling } & \multirow{2}{*}{$\begin{array}{l}\text { Months to } \\
\text { CSF } \\
\text { Involvement }\end{array}$} & \multirow{2}{*}{$\begin{array}{c}\text { Other Extranodal } \\
\text { Disease at Time of } \\
\text { Positive CSF }\end{array}$} \\
\hline & & & & $\begin{array}{c}\text { Clinical } \\
\text { Signs/Symptoms }\end{array}$ & Radiographic Lesion & & \\
\hline 1 & $78 / \mathrm{M}$ & I & Blastoid & Weakness, parasthesias & $\begin{array}{l}\text { Cerebropontine angle } \\
\text { mass }\end{array}$ & 21 & None \\
\hline 2 & $56 / \mathrm{M}$ & IV & Diffuse & Ptosis & $\begin{array}{l}\text { Abnormal meningeal } \\
\text { enhancement }\end{array}$ & 10 & $\begin{array}{l}\text { Testis, bone marrow, } \\
\text { peripheral blood }\end{array}$ \\
\hline 3 & $57 / \mathrm{F}$ & IV & Diffuse & $\begin{array}{l}\text { Headache, diplopia, } \\
\text { cranial nerve palsy }\end{array}$ & Unknown & 32 & $\begin{array}{l}\text { Bone marrow, } \\
\text { peripheral blood }\end{array}$ \\
\hline 4 & $44 / \mathrm{F}$ & IV & Diffuse & $\begin{array}{l}\text { Eye pain, ptosis, } \\
\text { diplopia, cranial } \\
\text { nerve palsy }\end{array}$ & None & 96 & Bone marrow \\
\hline 5 & $54 / \mathrm{M}$ & IV & Blastoid $^{a}$ & Mental status changes & Unknown & 29 & $\begin{array}{l}\text { Bone marrow, } \\
\text { peripheral blood }\end{array}$ \\
\hline 6 & $44 / \mathrm{M}$ & IV & Diffuse & $\begin{array}{l}\text { Headache, mental } \\
\text { status changes }\end{array}$ & $\begin{array}{l}\text { Abnormal meningeal } \\
\text { enhancement }\end{array}$ & 1 & Peripheral blood \\
\hline 7 & $63 / \mathrm{F}$ & IV & Diffuse & Weakness, ptosis & Diffuse spinal cord lesions & 6 & $\begin{array}{l}\text { Bone marrow, } \\
\text { peripheral blood }\end{array}$ \\
\hline 8 & $65 / \mathrm{M}$ & I & Diffuse & Mental status changes & Intracranial mass & 17 & Bone marrow \\
\hline 9 & $60 / \mathrm{M}$ & IV & Diffuse & Mental status changes & Unknown & 0 & $\begin{array}{l}\text { Breast, bone marrow, } \\
\text { peripheral blood }\end{array}$ \\
\hline 10 & $73 / \mathrm{F}$ & IV & Diffuse & $\begin{array}{l}\text { Weakness, mental } \\
\text { status changes }\end{array}$ & Unknown & 14 & Bone marrow \\
\hline
\end{tabular}

MCL, mantle cell lymphoma; CSF, cerebrospinal fluid.

${ }^{a}$ Diffuse histology at presentation with subsequent blastoid transformation in bone marrow. 
TABLE 3. CSF Characteristics of 10 Positive MCL Cases

\begin{tabular}{|c|c|c|c|c|c|c|c|}
\hline \multirow{2}{*}{$\begin{array}{l}\text { Patient } \\
\text { No. }\end{array}$} & \multicolumn{2}{|c|}{ Cell Count } & \multicolumn{2}{|c|}{ Chemistries } & \multirow{2}{*}{$\begin{array}{c}\% \\
\text { Lymphocytes }\end{array}$} & \multirow{2}{*}{$\begin{array}{c}\text { Flow } \\
\text { Cytometry }\end{array}$} & \multirow{2}{*}{$\begin{array}{c}\text { Leukemic } \\
\text { When CSF } \\
\text { Sampled }\end{array}$} \\
\hline & $\begin{array}{l}\text { Red Blood } \\
\text { Cells } / \mathrm{mm}^{3}\end{array}$ & $\begin{array}{l}\text { White Blood } \\
\text { Cells } / \mathrm{mm}^{3}\end{array}$ & $\begin{array}{l}\text { Protein } \\
(\mathrm{mg} / \mathrm{dL})^{a}\end{array}$ & $\begin{array}{l}\text { Glucose } \\
(\mathrm{mg} / \mathrm{dL})^{b}\end{array}$ & & & \\
\hline 1 & 250 & 4950 & 163 & 182 & 100 & Clonal & No \\
\hline 2 & 1900 & 1750 & 809 & 34 & 100 & Clonal & Yes \\
\hline 3 & 0 & 137 & 113 & 36 & 100 & Clonal & Yes \\
\hline 4 & 188 & 44 & 62 & 56 & 90 & Clonal & No \\
\hline 5 & 340 & 1010 & 255 & 34 & 100 & Clonal & Yes \\
\hline 6 & 2 & 5 & 34 & 83 & 96 & Clonal & Yes \\
\hline 7 & 3850 & 4850 & 120 & 34 & 88 & Clonal & Yes \\
\hline 8 & 46 & 197 & 98 & 78 & 98 & Not done & No \\
\hline 9 & 143 & 130 & 106 & 5 & 100 & Not done & Yes \\
\hline 10 & 311 & 1 & 28 & 64 & 68 & $\mathrm{QNS}^{c}$ & No \\
\hline
\end{tabular}

CSF, cerebrospinal fluid; MCL, mantle cell lymphoma.

${ }^{a}$ Normal range, $15-45 \mathrm{mg} / \mathrm{dl}$.

${ }^{b}$ Normal range, $50-70 \mathrm{mg} / \mathrm{dl}$.

${ }^{c}$ Quantity not sufficient for study.

tinctorial quality of the cytoplasm also varied; some cases contained neoplastic cells with abundant, eosinophilic cytoplasm, whereas others had cells with only scant blue-gray cytoplasm. Prominent cytoplasmic granules were observed in two of the three cases with immature, blastic cytologic features. These two cases included one with blastoid lymph node histology and one with typical lymph node histology (Fig. 3). Whereas the malignant cells in the CSF overall tended to retain the cytologic features of the diagnostic lymph node, the CSF in some cases contained a population of larger cells that were not characteristic of the lymph node at diagnosis.

Outcome data after CNS/CSF infiltration by MCL were not uniformly available for our cohort. In the three patients for whom follow-up information was available, however, CSF involvement by MCL portended a dismal prognosis, with two dying from persistent disease within 1 and 2 months (Cases 1 and 2, respectively) despite aggressive therapy including intrathecal methotrexate and radiation therapy. The third patient (Case 6) received an autologous bone marrow transplant shortly after diagnosis with MCL in the CSF but died 14 months later with relapsed disease.

\section{DISCUSSION}

MCL is an aggressive lymphoid malignancy with a poor prognosis. It remains incurable by standard therapy, and the overall median survival is reported to be 43 months. The relatively poor outcome observed in MCL patients can in part be attributed to the fact that the disease usually presents in advanced stage, frequently with multifocal disease including involvement at several extranodal sites. In a recent clinicopathologic study of 80 MCL patients, extranodal involvement at presentation was found in $76 \%$ of cases, and extranodal localization of dis- ease was the primary presentation in $25 \%$ of patients (4).

MCL may initially respond to combination chemotherapy like other histologically similar B-cell non-Hodgkin's lymphomas, but only a relatively small number of patients experience a complete response, and most relapse after only short diseasefree intervals. Despite this unfavorable clinical behavior and high relapse rate, current clinical staging protocols and evaluations of MCL patients do not routinely include assessment of the CSF as do those of other aggressive lymphoid malignancies such lymphoblastic and Burkitt-type lymphomas, which are known to frequently involve the CNS and for which prophylactic CNS therapy is administered. Moreover, only occasional reports specifically addressing the issue of CSF/CNS involvement in MCL appear in the literature (14-16).

To our knowledge, the first case of MCL involving the CNS was reported by Bedotto et al. in 1986 (16), and since then, relatively few additional authors have reported their experiences with CSF involvement in variably-sized cohorts of MCL patients. In 1996, Montserrat et al. (15) reported an approximately $23 \%$ incidence of CNS involvement in a cohort of 22 MCL patients followed for a median of 50 months. More recently, Oinonen et al. (14) studied 94 patients with MCL seen at one center over a 17 -year period and found CNS MCL in 4/94 (4\%) during a median follow-up of 51 months. The incidence of CSF involvement by MCL at presentation was reported to be approximately $2 \%$ by Meusers $e t$ $a l$. and Brittinger et al. (17-19) based on data from the Kiel Lymphoma Study Group.

In this study, we reviewed the clinical, radiographic, and pathologic records of 108 patients with well-characterized MCL to determine the frequency of CSF sampling and the rate of CSF involvement by MCL. Twenty-five patients in our cohort (23\%) underwent CSF sampling during the course of their 

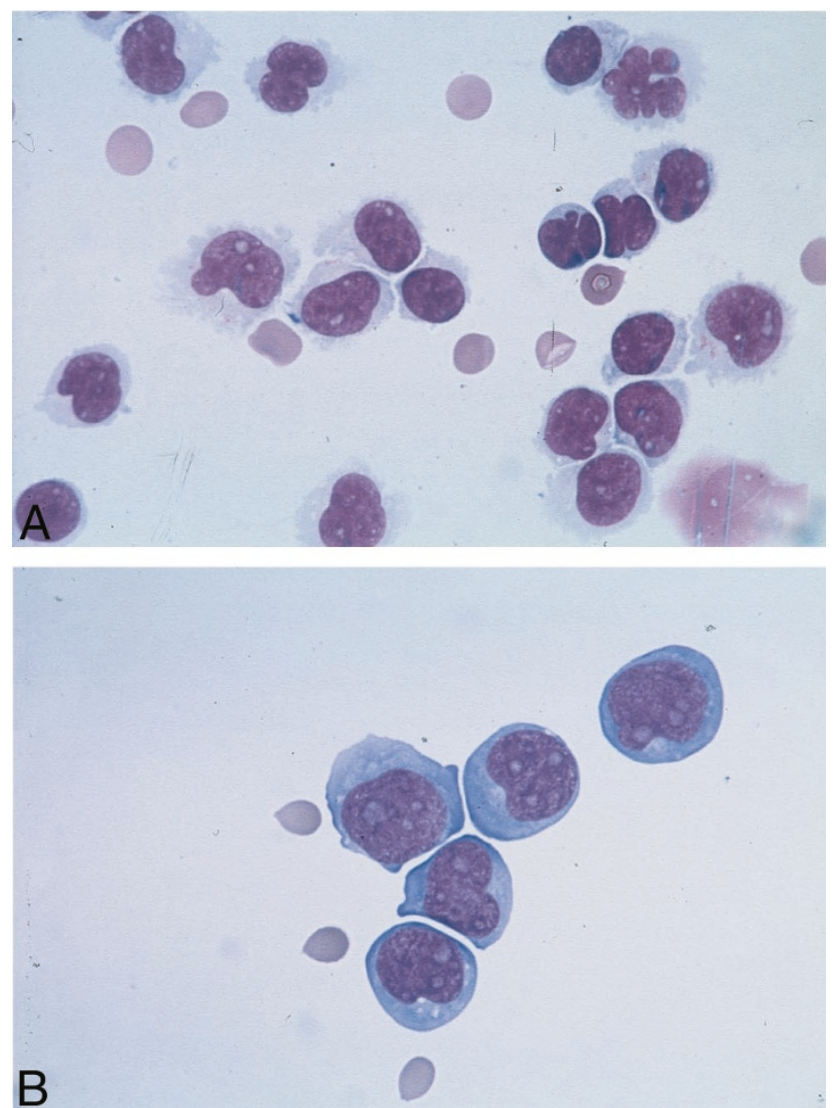

FIGURE 1. A-B, a pleomorphic population of lymphoid cells with irregular nuclear contours and variable amounts of cytoplasm was seen in the majority of cerebrospinal fluid-positive cases (Wright-stained cytocentrifuge preparation, high magnification).

disease. Lumbar punctures were performed as part of the evaluation of new-onset CNS signs and/or symptoms in all 25 patients. None of the patients had an LP before diagnosis with MCL, and none had CSF examinations as part of any routine or protocol-based staging work-up. The clinical indications for LP in our cohort were similar to those described for other patients suspected of having CNS lymphoma and included headache, mental status changes, and cranial nerve signs $(14,20,21)$.

CSF involvement by MCL was demonstrated in $40 \%(10 / 25)$ of the patients who underwent diagnostic LP for evaluation of CNS signs and/or symptoms, and CSF positivity was documented in $9 \%$ of the entire 108 MCL patient cohort. The overall rate of CSF involvement observed in our study differed from the $4 \%$ and $23 \%$ positivity rates published by other authors based on studies of equivalent patient cohorts with respect to demographics, clinical presentation, and stage data $(14,15)$. On the other hand, the overall positivity rate was comparable to that reported by Bosch et al. (7), who found CNS infiltration by MCL in $12 \%$ of a similar 59-patient cohort. As was the case with prior studies addressing the issue of CSF/CNS involvement in MCL, however, our findings also only provide a rough

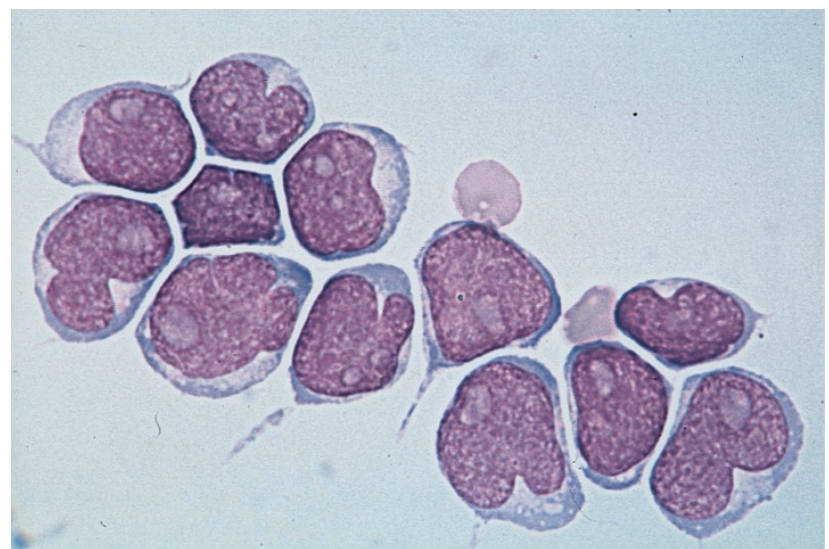

FIGURE 2. Large lymphoid cells with dispersed chromatin, prominent nucleoli, and scant cytoplasm resembling other blastic-type proliferations involving the cerebrospinal fluid were observed in the two cases of blastoid mantle cell lymphoma (MCL) as well as in one case of typical MCL (Wright-stained cytocentrifuge preparation, high magnification).

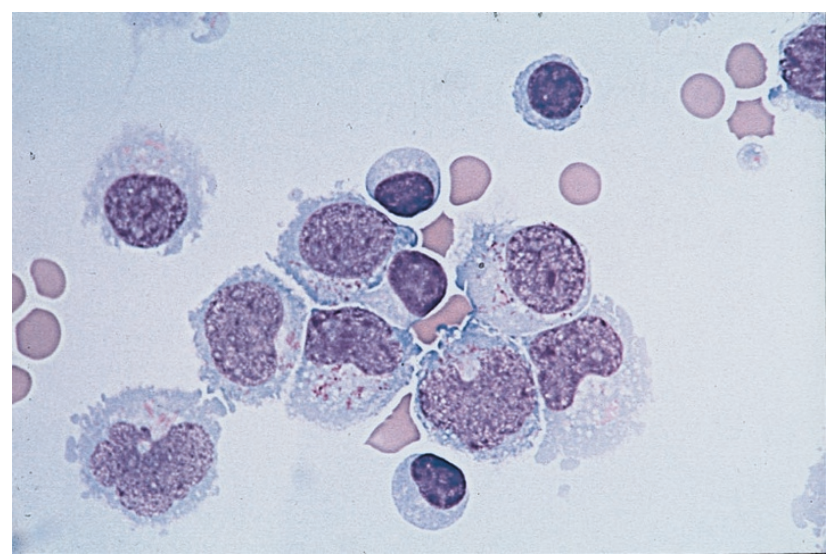

FIGURE 3. Prominent cytoplasmic granules were seen in two cases of mantle cell lymphoma involving the cerebrospinal fluid (Wrightstained cytocentrifuge preparation, high magnification).

estimate of the true incidence of CSF involvement by MCL because the CSF was not systematically or uniformly examined in a large majority $(77 \%)$ of the patients in our cohort. The actual incidence of CSF involvement by MCL during the course of disease is most likely underestimated in this as well as the other currently published series. Additional studies examining this issue will be necessary if and when an effective therapy for MCL is found and the role of CNS prophylaxis in MCL is questioned.

Although presentation with clinical CNS signs and/or symptoms prompted CSF examination in all cases, the presence of clinical findings alone did not appear to strongly predict CSF positivity because more than one half of the symptomatic patients had no morphologic or immunophenotypic evidence of CSF involvement despite multiple LPs. The presence of a radiographic abnormality in symptomatic MCL patients, however, did appear to correlate with lymphomatous involvement of the 
CSF because five of the six patients (83\%) with specific radiographic lesions had positive CSF samples. The demographic features and disease stage in our CSF-positive cohort were similar to those described in other studies of MCL patients with CNS/ CSF involvement. Of the 10 CSF-positive patients in our study, six were men, four were women, and the mean age at diagnosis with CNS lymphoma was 59.4 years (range, 22 to $78 \mathrm{y}$ ), which is slightly younger than the median age of the typical MCL patient $(63 \mathrm{y})$. The great majority of the CSFpositive patients $(80 \%)$ presented with advanced stage disease (Stage IV), with all Stage IV patients having bone marrow involvement and one half also having leukemic infiltrates in the peripheral blood at presentation. Two of the 10 CSF-positive patients, including one with blastoid MCL, presented with Stage I disease.

All patients developed CSF involvement within 32 months of diagnosis, with the exception of a single patient who developed CSF involvement at 96 months. The median time from initial diagnosis with MCL to CSF/CNS infiltration among our entire group of CSF-positive patients was 15.5 months (range, 0 to $96 \mathrm{mo}$ ). Given that the overall median survival of MCL patients is only 43 months, these findings on the length of time from initial diagnosis to CSF disease suggest that when MCL involves the $\mathrm{CSF} / \mathrm{CNS}$, it may be early in the course of disease or may even be present at the time of diagnosis (as seen in Case 9). This is in contrast to the findings of some previously published studies reporting that CSF involvement is a late complication of MCL (14). Interestingly, although both patients in the current study with the more aggressive blastoid variant of MCL developed CSF involvement, the median time to CSF positivity in these two cases $(25 \mathrm{mo})$ was longer than the median for the entire cohort. Irrespective of when MCL infiltrates the CNS, however, involvement of the CSF in MCL carries poor prognostic implications, as it does in other aggressive lymphomas.

Just as only a few authors have addressed the issue of CNS involvement in MCL, even fewer have attempted to characterize the cytomorphologic appearance of MCL cells in the CSF $(16,22)$. As such, another aim of our study was to specifically describe and illustrate the features of MCL cells as observed on the Wright-stained cytocentrifuge CSF smears that are routinely encountered in the hematology laboratory. Using such smears, a diagnosis of lymphomatous involvement of the CSF could be established on cytomorphologic grounds in 9 of the 10 cases. Too few cells for an adequate evaluation and definite morphologic diagnosis were present in 1 of the 10 cases; the diagnosis of MCL was established by flow cytometry alone in this case. Although we found that the appearance of MCL cells in the CSF was often variable, malignant lymphoma cells could be readily recognized morphologically in most cases because of their pleomorphic appearance, their similarity to blastic proliferations, and/or their occasional cytoplasmic granularity. This latter feature, which was also described in the first reported case of MCL in the CNS (16), is of unknown significance but is important to recognize as it may cause diagnostic confusion with acute myelogenous leukemia, especially in cases with prominent blastic cytology.

In summary, although we and a few other authors have reported our experiences with the rate of CSF involvement in MCL patients, the true incidence of this phenomenon is still not well defined. Other clinically aggressive lymphomas such as lymphoblastic and Burkitt-type lymphomas reportedly involve the CNS in 7-13\% of cases at diagnosis and in $16.5-39 \%$ during the course of disease $(23,24)$. However, because current clinical staging protocols and evaluations of MCL patients do not routinely include CSF examination as do the protocols for other aggressive lymphomas, the data needed to accurately characterize the true incidence of CSF involvement in MCL are not currently available. Given the findings of this and other studies, these data may prove to be important, especially if effective therapies for MCL become available. Moreover, because CSF involvement portends poor prognosis, pathologic recognition and confirmation of this complication are critical. Several nonneoplastic or reactive processes may result in CNS signs and/or symptoms and a CSF lymphocytosis in patients with lymphoma, and because immunophenotyping of CSF samples is not always possible, familiarity with the morphologic spectrum of MCL cells in routinely prepared Wright-stained cytocentrifuge CSF smears is important in recognizing lymphomatous involvement of the CSF.

\section{REFERENCES}

1. Rimokh R, Berger F, Delsol G, et al. Rearrangement and overexpression of the BCL-1/PRAD-1 gene in intermediate lymphocytic lymphomas and in $\mathrm{t}(1 \mathrm{qq} 13)$-bearing leukemias. Blood 1993;81:3063-7.

2. Weisenburger DD, Armitage JO. Mantle cell lymphoma-an entity comes of age. Blood 1996;87:4483-94.

3. Raffeld M, Sander CA, Yano T, et al. Mantle cell lymphoma: an update. Leuk Lymphoma 1992;8:161-6.

4. Argatoff LH, Connors JM, Klasa RJ, et al. Mantle cell lymphoma: a clinicopathologic study of 80 cases. Blood 1997;89: 2067-78.

5. Pittaluga S, Wlodarska I, Stul MS, et al. Mantle cell lymphoma: a clinicopathological study of 55 cases. Histopathology 1995;26:17-24.

6. Majlis A, Pugh WC, Rodriguez MA, et al. Mantle cell lymphoma: correlation of clinical outcome and biologic features with three histologic variants. J Clin Oncol 1997;15:1664-71. 
7. Bosch F, Lopez-Guillermo A, Campo E, et al. Mantle cell lymphoma: presenting features, response to therapy, and prognostic factors. Cancer 1998;82:567-75.

8. Tworek JA, Singleton TP, Schnitzer B, et al. Flow cytometric and immunohistochemical analysis of small lymphocytic lymphoma, mantle cell lymphoma, and plasmacytoid small lymphocytic lymphoma. Am J Clin Pathol 1998;110:582-9.

9. Huang JC, Finn WG, Goolsby CL, et al. CD5- small B-cell leukemias are rarely classifiable as chronic lymphocytic leukemia. Am J Clin Pathol 1999;111:123-30.

10. Douglas VK, Gordon LI, Goolsby CL, et al. Lymphoid aggregates in bone marrow mimic residual lymphoma after rituximab therapy for non-Hodgkin's lymphoma. Am J Clin Pathol 1999;112:844-53.

11. Maitra A, McKenna RW, Weinberg AG, et al. Precursor B-cell lymphoblastic lymphoma. A study of nine cases lacking blood and bone marrow involvement and review of the literature. Am J Clin Pathol 2001;115:868-75.

12. Kroft SH, Howard MS, Picker LJ, et al. De novo CD5-positive diffuse large B-cell lymphomas: a heterogeneous group containing an unusual form of splenic lymphoma. Am J Clin Pathol 2000;114:523-33.

13. Harris NL JE, Stein H, Banks PM, Chan JKC, Cleary ML, Delsol G, et al. A revised European-American classification of lymphoid neoplasms: a proposal from the International Lymphoma Study Group. Blood 1994;84:1361-92.

14. Oinonen R, Franssila K, Elonen E. Central nervous system involvement in patients with mantle cell lymphoma. Annu Hematol 1999;78:145-9.

15. Montserrat E, Bosch F, Lopez-Guillermo A, et al. CNS involvement in mantle-cell lymphoma. J Clin Oncol 1996;14:941-4.
16. Bedotto J, Spier CM, Paquin ML, et al. Mantle zone lymphoma with central nervous system involvement. Cancer 1986;58:2125-9.

17. Meusers P, Hense J, Brittinger G. Mantle cell lymphoma: diagnostic criteria, clinical aspects and therapeutic problems. Leukemia 1997;11:S60-4.

18. Brittinger $\mathrm{G}$, Bartels $\mathrm{H}$, Common $\mathrm{H}$, et al. Clinical and prognostic relevance of the Kiel classification of nonHodgkin's lymphomas: results of a prospective multicenter study by the Kiel Lymphoma Study Group. Hematol Oncol 1984;2:269-306.

19. Meusers P, Engelhard M, Bartels H, et al. Multicentre randomized therapeutic trial for advanced centrocytic lymphoma: anthracycline does not improve the prognosis. Hematol Oncol 1989;7:365-80.

20. MacKintosh FR, Colby TV, Podolsky WJ, et al. Central nervous system involvement in non-Hodgkin's lymphoma: an analysis of 105 cases. Cancer 1982;49:586-95.

21. Levitt LJ, Dawson DM, Rosenthal DS, et al. CNS involvement in the non-Hodgkin's lymphomas. Cancer 1980;45: 545-52.

22. Gagneten D, Hijaz YM, Jaffe ES, et al. Mantle cell lymphoma: a cytopathological and immunohistochemical study. Diagn Cytopathol 1996;14:32-7.

23. Sandlund JT, Murphy SB, Santana VM, et al. CNS involvement in children with newly diagnosed non-Hodgkin's lymphoma. J Clin Oncol 2000;18:3018-24.

24. Bollen EL, Brouwer RE, Hamers S, et al. Central nervous system relapse in non-Hodgkin lymphoma. A single-center study of 532 patients. Arch Neurol 1997;54:854-9. 